\title{
Dentists Knowledge and Attitude Towards Tobacco Cessation Counseling
}

\author{
Amber Kiyani ${ }^{1}$ \\ Rabia Ahmad ${ }^{2}$ \\ Maha Ahmad ${ }^{3}$ \\ Mahreen Tariq ${ }^{4}$
}

BDS, MS, FAAOMP, Dip-ABOMP

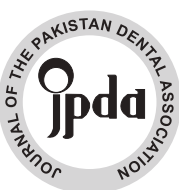

OBJECTIVE: Dentists play a critical role in identifying tobacco use in the oral cavity and counseling patients against the hazards associated with this habit. The objective of this study was to determine the current practices of dentists in Islamabad/Rawalpindi towards tobacco counseling, and their reasons, if any, for avoiding such counseling. METHODOLOGY: One hundred and five self- administered questionnaires were distributed in private dental clinics of Islamabad and Rawalpindi. The questions were designed to analyze tobacco history of dentists, their practices in acquiring appropriate histories, their skill in counseling, and reasons for deferring tobacco counseling. The information was recorded on SPSS version 20 and presented as frequencies and percentages.

Results: Out of the 105 questionnaires, only 97 were returned. About $81 \%(n=79)$ dentists asked male patients about tobacco use, only $48 \%(n=47)$ routinely asked female patients. Approximately $67 \%(n=65)$ dentists counsel their patients consistently, while $96 \%$ ( $\mathrm{n}=93$ ) counseled only after an oral pathology was discovered. Common reasons for avoiding counseling included belief that the smoking habit was the patient's business, 35.3\% ( $\mathrm{n}=23)$, fear of offending the patient $21.5 \%(\mathrm{n}=14)$, fear of negative impact on practice $1.5 \%(n=1)$ and the dentists own smoking habit $4.6 \%(n=3)$. Majority of the dentists were aware of nicotine replacement therapies but still believed that their background training was insufficient for counseling. CONCLUSION: The majority of Pakistani dentists have adequate knowledge and positive attitudes towards tobacco cessation counseling. Fear of intervening in patient's personal matter appears to be most common reason for not counseling. KEYWORDS: dentist, tobacco cessation counseling, NRT, tobacco cessation.

HOW TO CITE: Kiyani A, Ahmad R, Ahmad M, Tariq M. Dentists knowledge and attitude towards tobacco cessation counseling. J Pak Dent Assoc 2020;29(3):156-160.

DOI: https://doi.org/10.25301/JPDA.293.156

Received: 30 January 2020, Accepted: 12 May 2020

\section{INTRODUCTION}

$\mathrm{T}$ obacco is a recreational drug that contains a highly addictive substance called alkaloid nicotine. It is consumed as cigarettes, cigars, flavored shisha, snuff, chewing tobacco, dipping tobacco and snus. Despite its wide availability and use, tobacco is a documented health hazard that claims about 7 million lives yearly through cancer, pulmonary and cardiovascular diseases. ${ }^{1,2}$

In the oral cavity, tobacco consumption is linked to periodontal disease, delayed healing, treatment failures, staining of teeth, malodor, and oral cancer. ${ }^{2}$ Since patients with such problems see dentists first, they become crucial

1. Assistant Professor, Department of Oral Medicine, Riphah International University.

2. BDS Student, Islamic International Dental College (IIDC) Riphah.

3. BDS Student, Islamic International Dental College (IIDC) Riphah.

4. BDS Student, Islamic International Dental College (IIDC) Riphah.

Corresponding author: “Dr. Amber Kiyani ” < akiyani@gmail.com > for appropriately guiding patients against hazards of tobacco use. $^{3}$

Studies have shown that proper counseling about the hazards of smoking and information about cessation aids can encourage patients to quit. This can significantly improve patient outcome. ${ }^{4,5}$ Dental students at University of Buffalo, College of Dentistry performed protocol-based tobacco cessation counseling on 189 patients. During the 6-month follow-up survey, $32 \%$ of the sample had quit smoking and $22 \%$ had reduced the number of cigarettes they smoked per day. ${ }^{6}$ A Cochrane review published in 2012 demonstrated that increased quit rates can be achieved by dentists through active oral and video counseling. Similarly, Warnakulasuriya showed in 2002 that effective counseling by dentists can increase abstinence by $10-15 \%{ }^{7}$

Despite this, a prior study from Pakistan shows that only one-third of dentists are confident in their knowledge and skills to counsel patients against tobacco cessation. This 
study also determined that most dentists feel that tobacco cessation counseling falls outside their domain of responsibility. ${ }^{8}$ These results are backed by a study that asked patients if their dentist had ever talked to them about tobacco cessation, only $35 \%$ responded yes. ${ }^{9}$ However, results from another study performed later in 2016 had relatively encouraging results, where $80 \%$ of the dentists recognized their role in tobacco cessation counseling and over $70 \%$ would offer help to patients that were willing quit. ${ }^{10}$

Although there have been a few studies that explored the role of dentists in tobacco counseling in Pakistan, to the best of our knowledge, no study has offered reasons for dentists not providing tobacco cessation counseling. A study from the US found that lack of formal training in counseling, and no additional financial incentives for the effort were the most common reasons for avoiding involvement. Other reasons included patient reluctance to listen, extra time needed for counseling, and general lack of awareness. ${ }^{11}$

The aim of this investigation was to determine the knowledge and current practices of dentists in Islamabad towards smoking cessation counseling, and reasons for not offering it to their patients. This would help in prevention of tobacco related complications from occurring in these patients.

\section{METHODOLOGY}

This study was approved by the Ethical Review Board at Riphah International University (IIDC/IRC/2018/03/003).

A cross-sectional study was designed to evaluate the knowledge and attitude of dentists towards smoking cessation. A questionnaire was formulated following a thorough literature search to assess the current practices of dentists in tobacco counseling through four main themes; history taking practices, counseling practices, reasons for not counseling and prior formal training in counseling. The questions about history taking practices determined if the dentist routinely asked patients about smoking habits. The next section focused on the counseling practices of the dentists, whether the dentists counseled patients routinely or only when a pathology was identified. There was also a question about dentists introducing patients to nicotine replacement therapy (NRT). The third section determined the reasons for dentists for avoiding counseling. The last section identified the gaps in formal training in counseling. It also asked dentists whether the current dental curriculum was insufficient in training dentists in counseling.

This questionnaire was content validated by a community medicine expert, community dentist and a group of general dentists. The feedback from the reviewers was used to clarify some of the questions, and include questions that determined whether women were asked about their smoking habits, and the knowledge of dentists about NRT. This questionnaire was piloted on a group of 10 dentists. The reliability of the questionnaire was calculated through Cronbach alpha that produced a score of 0.71 .

The sample size was calculated using the WHO calculator. The population size of dentists was set as 4000 , population proportion $2 \%$, confidence interval $95 \%$ and margin of error $5 \%$. This produced a sample size of 30 .

The final questionnaire was distributed to 105 dentists across Islamabad between 15-01-2019 to 30-01-2019 through convenient sampling. This was done to ensure at least 100 forms would be returned. These self-administered questionnaires were only given to private practitioners across Islamabad/Rawalpindi. Since the project was meant to focus on community practice all dentists who were in private practice for more than 6 months in Islamabad/Rawalpindi region were invited to participate in the study. Dentists who worked in academic settings were excluded from the sample. This was done because academic institutes enforce better practices of history taking and counseling for teaching purposes so would produce better results. Dentists who were not working at the time of data collection were also excluded.

Consenting dentists were either given a brief overview of our investigation verbally, or in cases where they were short of time, a written statement with the same information was provided. The questionnaires were collected three days following distribution. The dentists who were unable to return the forms on our return visit were given a polite reminder and were visited three days later. The data was recorded on SPSS version 20. The software was used to calculate descriptive statistics for each category.

\section{RESULTS}

Out of the 105 questionnaires that were distributed to private dental practitioners in Islamabad and Rawalpindi, only 97 were returned, response rate $92 \%$. The practitioners consisted of predominantly general dentists and a few specialists. Their qualifications were not recorded.

From the 97 returned questionnaires, 49 (50\%) were from female dentists, 48 (50\%) were from males. Majority of the respondents were between the ages of 25-40 (mean $28.4+/-4.2)$. Thirteen (13.4\%) admitted to being smokers, $37(38 \%)$ left the question blank while $47(48 \%)$ were non-smokers.

While taking social history, $70(81 \%)$ dentists ask their male patients about tobacco use, while 18 (19\%) did not. In comparison, only $47(48 \%)$ routinely asked female patients 
about their tobacco history. As a reason for not asking female patients about their smoking habits,

Sixty-five $(67 \%)$ dentists would counsel patients against the hazards of smoking even when no pre-neoplastic or neoplastic disease was identified. Ninety-six percent $(n=93)$ dentists would offer some form of counseling if such diseases were identified. The summary of results is highlighted in Table 1.

Dentists who avoided counseling patients against their tobacco habits, the frequently cited reason for their practice

Table 1: Responses of participating dentists to our questionnaire $(\mathrm{N}=97)$

\begin{tabular}{|l|l|l|}
\hline Frequency of distribution of responses: & YES & NO \\
\hline Smokers & $13(13.4 \%)$ & $84(87 \%)$ \\
\hline Ask male patients about tobacco & $79(81 \%)$ & $18(19 \%)$ \\
\hline Ask female patients about tobacco & $47(48 \%)$ & $50(52 \%)$ \\
\hline Advises routinely & $65(67 \%)$ & $32(33 \%)$ \\
\hline Advises when pathology detected & $93(96 \%)$ & $4(4.1 \%)$ \\
\hline Knowledge about NRT & $69(71 \%)$ & $28(29 \%)$ \\
\hline Offers NRT to patients & $36(37 \%)$ & $61(63 \%)$ \\
\hline Confident in counseling & $40(41 \%)$ & $57(59 \%)$ \\
\hline Advocate reforms in curriculum for TCC & $89(92 \%)$ & $8(8 \%)$ \\
\hline
\end{tabular}

was that they believed that advising was interfering in patient's personal business, this included 23 (35.3\%) dentists. This was followed by a fear of offending the patient, 14 (21.5\%). Less frequently cited reasons included fear of a negative impact on practice, 1 (1.5\%), and dentist's smoking habit, $3(4.6 \%)$. Common reasons for not counseling are highlighted in Table 2.

Seventy-nine (71\%) dentists were aware of nicotine

Table 2: Responses of participants for reasons of avoiding smoking cessation counseling in their pratices. $(\mathrm{N}=65)$

\begin{tabular}{|l|l|l|}
\hline Reasons for avoiding counseling & YES & NO \\
\hline Dentist is a smoker & $3(4.6 \%)$ & $62(95 \%)$ \\
\hline Fear of offending & $14(21.5 \%)$ & $51(78 \%)$ \\
\hline Fear of negative impact on practice & $1(1.5 \%)$ & $64(98.4 \%)$ \\
\hline Belief that it is patient's business & $23(35.3 \%)$ & $42(64.6 \%)$ \\
\hline
\end{tabular}

replacement therapies available in the market. However, only $36(37 \%)$ dentists offered them as possible replacements to tobacco consumption during counseling. Majority of the dentists were aware of nicotine patches and gum. Less were familiar with medications and e-cigarettes.

Forty (40\%) dentists believed that their dental training was sufficient in the aspect of tobacco counseling, however, 89 (92)\% agreed that the curriculum should be modified to train students in addressing these issues with their patients.

\section{DISCUSSION}

Tobacco use is a significant risk factor for development of oral premalignancy and malignancy. ${ }^{12}$ Patient counseling about the hazards of tobacco use by dentists can encourage patients to quit. This investigation aimed to determine the knowledge and current practices of Pakistani dentists in tobacco cessation counseling and the reasons for not offering it.

There were $81.4 \%$ dentists in our sample that routinely asked male patients about their tobacco habits. However, only $48.4 \%$ asked females. The participants assumed that females don't smoke because of the low prevalence of female tobacco users in the country. Dentists were also hesitant because they were afraid of offending females. Although our results are encouraging when compared with an Australian survey in which only $14 \%$ dentists asked patients about their tobacco habits despite over $70 \%$ believing that it was their moral responsibility to counsel patients, they present an interesting gender-based discrepancy exclusive to our region. ${ }^{13}$

The prevalence of smokers in our sample was lower than previously reported in Pakistani dentists. We had only $13.4 \%$ smokers compared with $20.3 \%$ reported by Mumtaz in $2008 .^{8}$ However, we had a significant number of participants that refused to answer the question. So it is difficult for us to estimate the prevalence of dentists who smoke. This information may have been kept from us due to embarrassment reasons.

Our study shows that $95.8 \%$ dentists counseled patients against tobacco use when a significant oral pathology was present. However, only $67 \%$ offered counseling in the absence of any oral disease. Our results are relatively better than those produced by an English study where $71 \%$ dentists counseled patients at a significant risk for oral cancer, while only $60 \%$ counseled patients with other smoking-related oral issues. ${ }^{14}$ While encouraging, we believe that this discrepancy results from our data collection in the IslamabadRawalpindi region. The dentists here have better access to continuing education opportunities, hence are better aware. A similar study in the peripheral region would have produced different results.

Studies show that graduates with less than 10 years of practice tend to be more vigilant in acquiring thorough social history and in tobacco cessation counseling when compared with older graduates. ${ }^{15}$ This appears consistent with our results as the majority of our participants had a mean age of 28.4. Similar to reduction in the prevalence of tobacco using dentists, this can be attributed to better awareness about the harmful effects of smoking in recent decades. Dentists trained several decades ago would be deficient in such knowledge.

The most common reason for not offering tobacco cessation counseling in our sample was fear of interfering in the patient's business. Other studies have listed lack of 
time, inadequate formal training in tobacco cessation counseling, fear of losing patient and lack of financial incentive as possible causes for not counseling. ${ }^{16,17}$

In our study, $71 \%$ practicing dentists in our study were aware of replacement therapies available, however, this awareness was limited to nicotine patches and gums. The knowledge about medications, e-cigarettes, nasal sprays, vape, vaporizers, electronic devices and behavioral therapy centers was deficient. Only $37 \%$ dentists offered NRT to their patients. A study showed that despite positive attitude of post-graduate dental students towards NRT, only $34 \%$ had offered it as a smoking cessation aid. Compliance issues and cost were significant deterrents for these students. ${ }^{18}$ This may be an important consideration for our dentists as well while offering such solutions to their patients.

Our study indicated that $59 \%$ dentists did not feel competent in tobacco cessation counseling. A survey carried out by the Canadian Dental Hygienists Association revealed that $44 \%$ of dental professionals restrained themselves from advising their patients about tobacco cessation because they believe that they do not have enough knowledge, or sufficient training to guide patients. ${ }^{19}$

About $92 \%$ of the respondents in our study believed that the dental curriculum should be modified to include training for smoking cessation. This opinion is common in other studies..$^{20,21}$ Although our curriculum incorporates the biological effects of tobacco use, tobacco culture and psychosocial aspects of tobacco use, we need to introduce knowledge of prevention and treatment of tobacco use and dependence, and clinical training in cessation counseling as recommended by the consensus report of first European workshop on tobacco use and prevention. ${ }^{21}$ Introducing these themes into the curriculum would make our graduates more confident in offering help to their patients.

Since the data was collected from a single city and an urban setting, our sample is not reflective of the entire Pakistani dentist population. Knowledge and practices of dentists working in the periphery would be very different than we are currently reporting. A stronger study would need a larger sample size, inclusion of dentists from private and government-based practices from urban and rural settings across Pakistan.

\section{CONCLUSION}

The majority of Pakistani dentists have adequate knowledge and positive attitudes towards tobacco cessation counseling. Fear of intervening in patient's personal business is the most common cause for avoidance of counseling. Formal trainings in cessation aids and counseling can make dentists more confident in offering help to tobacco users .

\section{CONFLICT OF INTEREST}

None declared

\section{REFERENCES}

1. WHO. Tobacco. In: WHO, (ed.). 2018.

2. Omana-Cepeda C, Jane-Salas E, Estrugo-Devesa A, ChimenosKustner E and Lopez-Lopez J. Effectiveness of dentist's intervention in smoking cessation: A review. J Clin Exp Dent. 2016;8:e78-83. https://doi.org/10.4317/jced.52693

3. Awan KH, Hammam MK and Warnakulasuriya S. Knowledge and attitude of tobacco use and cessation among dental professionals. Saudi Dent J. 2015;27:99-104.

https://doi.org/10.1016/j.sdentj.2014.11.004

4. Gordon JS, Lichtenstein E, Severson HH and Andrews JA. Tobacco cessation in dental settings: research findings and future directions. Drug Alcohol Rev. 2006;25:27-37.

https://doi.org/10.1080/09595230500459495

5. Ehizele AO, Azodo CC, Ezeja EB and Ehigiator O. Nigerian dental students' compliance with the 4As approach to tobacco cessation. J Prev Med Hyg. 2011;52:12-6.

6. Shibly O. Effect of tobacco counseling by dental students on patient quitting rate. J Dent Educ. 2010;74:140-48.

7. Warnakulasuriya S. Effectiveness of tobacco counseling in the dental office. J Dent Educ. 2002; 66:1079-87.

8. Mumtaz R, Khan AA, Moeen F, Noor N and Humayun S. The role of Pakistani dentists in tobacco cessation. Int Dent J. 2008;58: 56-62. https://doi.org/10.1111/j.1875-595X.2008.tb00357.x

9. Khattak A AA, Khan SS, Irshad M. Role of dentist in tobacco cessation: A cross-sectional survey. Pak Oral Dent J. 2015;35:4.

10. Farrukh U SS, Nisar s. Dentists' practice and perceived barriers towards smoking cessation and Intervention in Karachi, Pakistan. J Pharma Care Health Sys. 2016;3.

https://doi.org/10.4172/2376-0419.1000151

11. Albert D, Ward A, Ahluwalia K and Sadowsky D. Addressing tobacco in managed care: a survey of dentists' knowledge, attitudes, and behaviors. Am J Public Health. 2002;92:997-1001. https://doi.org/10.2105/AJPH.92.6.997

12. Moreno-Lopez LA, Esparza-Gomez GC, Gonzalez-Navarro A, Cerero-Lapiedra R, Gonzalez-Hernandez MJ and Dominguez-Rojas V. Risk of oral cancer associated with tobacco smoking, alcohol consumption and oral hygiene: a case-control study in Madrid, Spain. Oral Oncol. 2000;36:170-74.

https://doi.org/10.1016/S1368-8375(99)00084-6

13. Rikard-Bell G and Ward J. Australian dentists' educational needs for smoking cessation counseling. J Cancer Educ. 2001; 16: 80-4. 
14. John JH, Thomas D and Richards D. Smoking cessation interventions in the Oxford region: changes in dentists' attitudes and reported practices 1996-2001. Br Dent J. 2003; 195: 270-5; discussion 61.

https://doi.org/10.1038/sj.bdj.4810480

15. Albert DA, Severson H, Gordon J, Ward A, Andrews J and Sadowsky D. Tobacco attitudes, practices, and behaviors: a survey of dentists participating in managed care. Nicotine Tob Res. 2005; 7 Suppl 1: S9-18.

https://doi.org/10.1080/14622200500078014

16. George B, Mulamoottil V, Cherian S, John J, Mathew T and Sebastian S. Awareness on smoking cessation counseling among dentists in Kerala, India. J Indian Assoc Publ Health Dentis. 2015;13:254-58.

https://doi.org/10.4103/2319-5932.165245

17. Li KW and Chao DV. Current practices, attitudes, and perceived barriers for treating smokers by Hong Kong dentists. Hong Kong Medi $\mathrm{J}=$ Xianggang yi xue za zhi. 2014; 20: 94-101.

https://doi.org/10.12809/hkmj134027
18. Shah S, Rath H and Sharma G. Knowledge, attitude and practices of institution-based dentists toward nicotine replacement therapy. Indian J Dent Res. 2017; 28:629-36. https://doi.org/10.4103/ijdr.IJDR_231_17

19. Association CDH. Tobacco Use Cessation Services and the Role of the Dental Hygienist- A CDHA position paper. Can J Dent Hygiene. 2004;38:260-79.

20. Crail J, Lahtinen A, Beck-Mannagetta J, et al. Role and models for compensation of tobacco use prevention and cessation by oral health professionals. Int Dent J. 2010;60:73-9.

21. Ramseier CA, Mattheos N, Needleman I, Watt R and Wickholm S. Consensus report: First European Workshop on Tobacco Use Prevention and Cessation for Oral Health Professionals. Oral Health Prevent Dentis. 2006;4:7-18. 